\title{
Estado del conocimiento sobre la sal de Friedel
}

\section{Condition of knowledge about the Friedel's salt}

\author{
R.LANNEGRAND, G.RAMOSYR.TALERO \\ Instituto de Ciencias de la Construcción Eduardo Torroja (CSIC)
}

Fecha de recepción: : 20-VII-00

Fecha de aceptación: 16-XI-00

ESPAÑA

\section{RESUMEN}

Se ha realizado un extenso estudio bibliográfico sobre la sal de Friedel. Se indica su estructura, sus probables mecanismos de formación e intervalos de estabilidad en presencia de otras sales, asi como también el efecto de la temperatura y el $\mathrm{pH}$.

\section{INTRODUCCIÓN}

La sal de Friedel debe su nombre a que en 1897, Friedel (1) sintetizó el monocloroaluminato cálcico hidratado, $3 \mathrm{CaO} \mathrm{Al} \mathrm{O}_{3} \mathrm{CaCl}_{2} \mathrm{Al}_{2} \mathrm{O}_{3} 10 \mathrm{H}_{2} \mathrm{O}$. Esta sal de Friedel puede encontrase en una pasta de cemento, cuando dicha pasta está próxima a una fuente de cloruros. Las principales fuentes de cloruros son: las que provienen de echar $\mathrm{NaCl}$ $\mathrm{y} / \mathrm{o} \mathrm{CaCl}{ }_{2}$ a las carreteras de hormigón para mantenerlas libres de hielo y nieve durante los meses de invierno en las regiones y/o países fríos; las que provienen de las fábricas de salazones y pescados; las que provienen de las aguas y ambiente marino en las zonas costeras y las que provienen del $\mathrm{CaCl}_{2}$ utilizado como acelerador del fraguado.

La capacidad de fijación de los cloruros por el cemento depende de la naturaleza y composición de este último, de la cantidad de aluminato tricálcico presente en el clínker, de la sal clorada empleada, de la relación aguacemento, del tiempo de curado, etc. (2-13). Además, el ferrito-aluminato tetracálcico $\mathrm{C}_{4} \mathrm{AF}$, también, es susceptible de formar la sal clorada análoga a la sal de Friedel, $3 \mathrm{CaO} \mathrm{Fe}_{2} \mathrm{O}_{3} \mathrm{CaCl}_{2} 10 \mathrm{H}_{2} \mathrm{O}(2,14)$. Sin embargo, $y$ al igual que ocurre con los sulfatos, el $\mathrm{C}_{3} \mathrm{~A}$ reacciona más rápidamente co n lo $\mathrm{s}$ cloruros que el $\mathrm{C}_{4} \mathrm{AF}$, por lo

\section{SUMMARY}

A vast bibliography revision is done about the Friedel's salt. The structure, more possible mechanisms of formation and stability intervals in presence of other salts and temperature effect and by pH are pointed out. que la mayoría de los complejos clorados inicialmente formados en un OPC hidratado son sal de Friedel, de origen $\mathrm{C}_{3} \mathrm{~A}$.

La sal de Friedel no sólo se puede formar en un cemento Portland ordinario sino que los iones cloruro se pueden enlazar también al aluminio de una escoria granulada de alto horno (15) y al aluminio de la alúmina reactiva, vítra o amorfa de una puzolana y/o ceniza volante (16).

La presencia de otras especies aniónicas en el sistema pasta de cemento-ambiente afecta a la capacidad de fijación química de los iones cloruros a las fases alumínicas (17-19).

A continuación se pasa a describir el estado del conocimiento sobre la sal de Fricdel, en aspectos relativos a su estructura, mecanismos de formación, estabilidad y caracterización.

\section{ESTRUCTURA DE LA SAL DE FRIEDEL}

La sal de Fricdel y varios aluminatos y ferritos hidratados relacionados con él, pertenecen a la familia de los hidratos tipo $\operatorname{AFm}(5,20)$. Los hidratos AFm tienen una estructura 
en placas hexagonales apiladas parecidos a la estructura cristalina de la portlandita, $\mathrm{Ca}(\mathrm{OH})_{2}$. La portlandita está formada por una red en placas apiladas en la que cada ion calcio, $\mathrm{Ca}^{2+}$, cstá rodeado por seis iones hidroxilo, $\mathrm{OH}^{-}$, en coordinación octaédrica (Fig. 1). Cada ion $\mathrm{OH}^{-}$está coordinado además de con $\mathrm{cl}$ ion $\mathrm{Ca}^{2+}$ central, con dos iones $\mathrm{Ca}^{2+}$ de capas adyacentes (cada oxígeno está, por tanto, en coordinación tetraédrica). La formación de la sal de Friedel la podemos imaginar como una sustitución ordenada de un ion $\mathrm{Ca}^{2+}$ de cada tres, en una capa de $\mathrm{Ca}(\mathrm{OH})_{2}$, por un ion $\mathrm{Al}^{3+}$. La razón de esta sustitución no esta clara, sin embargo, los átomos de $\mathrm{Al}$ son más pequeños en tamaño que los átomos de $\mathrm{Ca} y$, por tanto, estabilizarían la estructura del cristal. Como resultado de la sustitución se produce un desequilibrio de cargas en la "capa principal", $\left[\mathrm{Ca}_{2} \mathrm{Al}\left(\mathrm{OH}^{-}\right)_{6} 2 \mathrm{H}_{2} \mathrm{O}\right]^{+}$. Para compensar la carga positiva, un ion $\mathrm{Cl}^{-}$(en sentido figurado) se colocaría por encima del $\mathrm{Al}^{3+}$ en el hucco que hay entre los iones $\mathrm{OH}^{-}$(Fig. 2). Entonces, la composición de la capa principal se transformaría en $\left[\mathrm{Ca}_{2} \mathrm{Al}\left(\mathrm{OH}^{-}\right)_{6} \mathrm{H}_{2} \mathrm{O}\right] \mathrm{Cl}$. La celda unidad de la sal de Friedel consiste, por tanto, en dos capas principales idénticas de la composición anterior.

Existen dos modificaciones de la sal de Friedel (21) dependiendo de la temperatura, una por debajo de $30^{\circ} \mathrm{C}$ (monoclínico) y otra por encima (romboédrico) que se pueden distinguir por DRX (el valor de 2-theta varía de

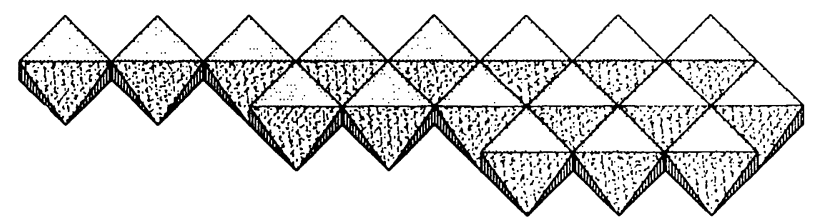

Figura 1.- Estructura cristalina del $\mathrm{Ca}(\mathrm{OH})_{2}$; disposición de los octaedros $\mathrm{Ca}(\mathrm{OH})_{6}$ enlazados dentro de una misma capa.

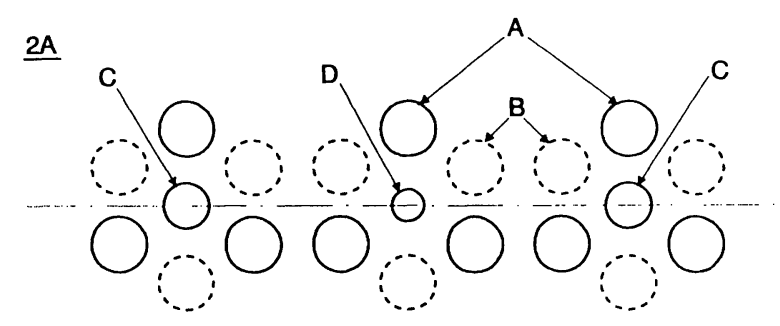

$\underline{2 B}$

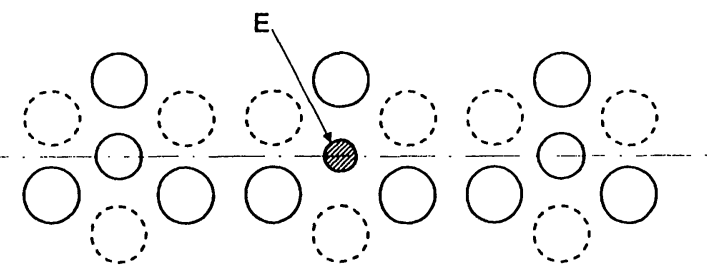

Figura 2.- (2A): vista esquemática de la capa principal AFm. (2B): adsorción de un ion $\mathrm{Cl}^{-}$sobre el $\mathrm{Al}^{3+}$ en el hueco existente entre los iones $\mathrm{OH}$. Notación: A: iones $\mathrm{OH}^{-}$en la capa superior. $\mathrm{B}$ : iones $\mathrm{OH}^{-}$en la capa inferior. $\mathrm{C}$ : ion $\mathrm{Ca}^{2+}$. D: ion $\mathrm{Al}^{3+}$. E: ion $\mathrm{Cl}^{-}(20)$. $11,24^{\circ}$ a $11,33^{\circ}$ cuando pasamos de la fase de baja a la de alta temperatura). La transición de una a otra es casi instantánea, reversible y produce una disminución tanto de volumen, del 2,6\%, como del espaciado de las intercapas, de $7,87 \AA$ a $7,81 \AA$, al pasar de la fase de baja temperatura a la de alta.

\section{MECANISMO DE FORMACIÓN DE LA SAL DE FRIEDEL}

Suryavanshi y col. (20) observaron que a medida que el ion cloruro se va fijando a los aluminatos de un cemento rico en $\mathrm{C}_{3} \mathrm{~A}$, se produce un aumento en la concentración del ion $\mathrm{OH}^{-}$en la disolución del poro. Sin embargo, la cantidad de iones $\mathrm{OH}^{-}$liberados a la disolución del poro no es equivalente a la cantidad de cloruro fijado durante la hidratación. Esto sugierc que los iones libres de cloruro se enlazan simultáneamente con los aluminatos del cemento mediante dos mecanismos diferentes. Una fracción de los iones libres de cloruro se enlazarían liberando una cantidad equivalente en moles de iones $\mathrm{OH}^{-}$dentro de la disolución del poro, mientras que la mayoría de los iones libres de cloruro se enlazarían sin que ocurra una liberación de iones $\mathrm{OH}$ - hacia la disolución del poro. Ambos mecanismos se explican a continuación separadamente.

\section{Mecanismo de enlace del ion $\mathrm{Cl}^{-}$con liberación de ion $\mathrm{OH}^{-}$}

Ben-Yair (22) explica con dos ecuaciones, cl aumento de la concentración del ion $\mathrm{OH}^{-}$producido por el enlace de los iones cloruro cuando se añade a la mezcla $\mathrm{NaCl}$.

(a) $\mathrm{Ca}(\mathrm{OH})_{2}+2 \mathrm{NaCl} \rightleftharpoons \mathrm{CaCl}_{2}+2 \mathrm{Na}^{+}+2 \mathrm{OH}^{-}$

(b) $\mathrm{C}_{3} \mathrm{~A}+\mathrm{CaCl}_{2}+10 \mathrm{H}_{2} \mathrm{O} \rightarrow \mathrm{C}_{3} \mathrm{~A} \cdot \mathrm{CaCl}_{2} \cdot 10 \mathrm{H}_{2} \mathrm{O}$

El consumo de $\mathrm{CaCl}_{2}$ según la reacción (b) desplazaría el equilibrio de la ecuación (a) hacia la derecha y causaría la disolución de la portlandita liberando iones hidroxilo a la disolución del poro. Sin embargo, la formación de la sal de Friedel según la ecuación (b) está abicrta a debate debido a la alta solubilidad del $\mathrm{CaCl}_{2}$ incluso en disoluciones fuertemente alcalinas. Además, la formación de la sal de Friedel según la ecuación (b) es dificil de explicar atendiendo a su estructura.

Alternativamente a la hipótesis anterior, este aumento en la concentración del ion $\mathrm{OH}$, puede ser explicado también por una sustitución ordenada de iones $\mathrm{OH}^{-}$, presentes en las intercapas de los hidratos $\mathrm{AFm}\left(\mathrm{C}_{4} \mathrm{AH}_{13}\right.$ y sus derivados), por los $\mathrm{Cl}^{-} \mathrm{de}$ la disolución del poro.

(c) $3 \mathrm{CaO} \cdot \mathrm{Al}_{2} \mathrm{O}_{3}+\mathrm{Ca}(\mathrm{OH})_{2}+12 \mathrm{H}_{2} \mathrm{O} \rightarrow 4 \mathrm{CaO} \cdot \mathrm{Al}_{2} \mathrm{O}_{3} \cdot \mathrm{H}_{2} \mathrm{O}$ 
La fase $\mathrm{C}_{4} \mathrm{AH}_{13}$ es meta-estable y puede conllevar cambios en el contenido de agua para formar otros hidratos AFm con mayor/menor contenido en moléculas de agua en la capa principal. Los iones $\mathrm{OH}^{-}$de las intercapas son especialmente propensos al intercambio iónico. De este modo, la reacción de intercambio aniónico ocurriría entre estos iones $\mathrm{OH}^{-}$de las intercapas de los hidratos AFm y los iones cloruro libres de la disolución del poro para formar la sal de Friedel como sigue:

$$
\text { (d) } \mathrm{R}-\mathrm{OH}^{-}+\mathrm{Na}^{+}+\mathrm{Cl}^{-} \rightarrow \mathrm{R}-\mathrm{Cl}^{-}+\mathrm{Na}^{+}+\mathrm{OH}^{-}
$$

donde, "R" es la capa principal de los hidratos AFm de composición $\left[\mathrm{Ca}_{2} \mathrm{Al}\left(\mathrm{OH}^{-}\right)_{6} \mathrm{nH}_{2} \mathrm{O}\right]^{+}$y el valor de " $\mathrm{n}$ " depende del tipo de hidrato AFm que participe en la reacción. Así, el aumento en moles de $\mathrm{OH}^{-}$que ocurre durante la fijación de los cloruros es equivalente a los moles de iones cloruro enlazados. La cantidad de iones $\mathrm{OH}^{-}$que se intercambiarían con los $\mathrm{Cl}^{-}$dependería, por tanto, del númcro de posiciones posibles para el intercambio ionico, o indirectamente, de la cantidad de hidratos $\mathrm{AFm}$ presentes en determinado momento en el cemento hidratado. Por lo que según esta explicación, el límite máximo de intercambio iónico puede ser interpretado como la capacidad de intercambio iónico de los hidratos AFm.

\section{Mecanismo de enlace del ion $\mathrm{Cl}^{-}$sin liberación de $\mathrm{OH}^{-}$}

De la discusión anterior, parece claro que una fracción del total de los iones cloruro libres presentes en la disolución del poro se enlazan mediante un mecanismo de intercambio aniónico. Sin embargo, los datos experimentales indican que csto sólo responde para una pequeña fracción de los $\mathrm{Cl}^{-}$enlazados. La mayoría de los iones cloruro libres, debidos al $\mathrm{NaCl}$ y/o al $\mathrm{CaCl}_{2}$, se enlazan mediante una adsorción directa a las intercapas de la capa principal, $\left[\mathrm{Ca}_{2} \mathrm{Al}\left(\mathrm{OH}^{-}\right)_{6} 2 \mathrm{H}_{2} \mathrm{O}\right]^{+}$, de la estructura AFm para compensar el exceso de carga positiva, debida a la sustitución de un ion $\mathrm{Ca}^{2+}$ porun ion $\mathrm{Al}^{3+}$. La adsorción de iones $\mathrm{Cl}^{-}$, conduce a la formación de la sal de Friedel tal y como ya se ha explicado y altera el balance de carga iónica en la disolución del poro. Para mantener la neutralidad de cargas, una cantidad equivalente de iones $\mathrm{Na}^{+}$dejaría la disolución del poro y entraría en la red del gel CSH, como se explica a continuación:

Cationes como el $\mathrm{Al}^{3+}$ y el $\mathrm{Fe}^{3+}$ pueden sustituir al $\mathrm{Si}^{4+} \mathrm{de}$ la estructura del gel CSH y, como resultado de ésto y para compensar la carga, cationes alcalinos monovalentes, iones $\mathrm{K}^{+}$y $\mathrm{Na}^{+}$, entrarían en la estructura del gel CSH junto con iones $\mathrm{Ca}^{2+}$. El límite superior de tal sustitución es un átomo de $\mathrm{Al}$ o $\mathrm{Fe}$ por seis átomos de $\mathrm{Si}$ (23).

\section{ESTABILIDAD DE LA SAL DE FRIEDEL}

\section{I El Sistema $\mathrm{CaO}-\mathrm{Al}_{2} \mathrm{O}_{3}-\mathrm{CaCl}_{2}-\mathrm{H}_{2} \mathrm{O}$. Influencia del $\mathrm{Na}_{2} \mathrm{O}$}

En 1994, Damidot y col. (18) calcularon el diagrama de equilibrio de fases del sistema $\mathrm{CaO}-\mathrm{Al}_{2} \mathrm{O}_{3}-\mathrm{CaCl}_{2}-\mathrm{H}_{2} \mathrm{O}$ a $25^{\circ} \mathrm{C}$ y encontraron la presencia de 6 puntos invariantes:

$\mathrm{CH}+\mathrm{C}_{3} \mathrm{AH}_{6}+$ monocloroaluminato cálcico hidratado.

$\mathrm{AH}_{3}+\mathrm{C}_{3} \mathrm{AH}_{6}+$ monocloroaluminato cálcico hidratado.

$\mathrm{CH}+3 \mathrm{CaO} \cdot \mathrm{CaCl}_{2} \cdot 15 \mathrm{H}_{2} \mathrm{O}+$ monocloroaluminato cálcico hidratado.

$3 \mathrm{CaO} \cdot \mathrm{CaCl}_{2} \cdot 15 \mathrm{H}_{2} \mathrm{O}+\mathrm{CaO} \cdot \mathrm{CaCl}_{2} \cdot 2 \mathrm{H}_{2} \mathrm{O}+$ monocloroaluminato cálcico hidratado.

$\mathrm{CaCl}_{2} \cdot 6 \mathrm{H}_{2} \mathrm{O}+\mathrm{AH}_{3}+$ monocloroaluminato cálcico hidratado.

$\mathrm{CaO} \cdot \mathrm{CaCl}_{2} \cdot 2 \mathrm{H}_{2} \mathrm{O}+\mathrm{CaCl}_{2} \cdot 6 \mathrm{H}_{2} \mathrm{O}+$ monoclorualuminato cálcico hidratado.

El pKs (24) de la sal de Fricdel es 27.10. El monocloroaluminato cálcico hidratado es estable en un amplio intervalo de concentración de cloruros $\left(0,0096<\left[\mathrm{Cl}^{-}\right]<8,016 \mathrm{~mol} / \mathrm{kg}\right)$, lo que está en consonancia con Birnin-Yauri y col. $(24)\left(\sim 0,0145<\left[\mathrm{Cl}^{-}\right]<\sim 8 \mathrm{M}\right)$. De la representación gráfica tridimensional del sistema (Fig. 3), Damidot y col. sugiricron que la sal de Friedel debería ser estable en zonas degradadas de la pasta de cemento ya

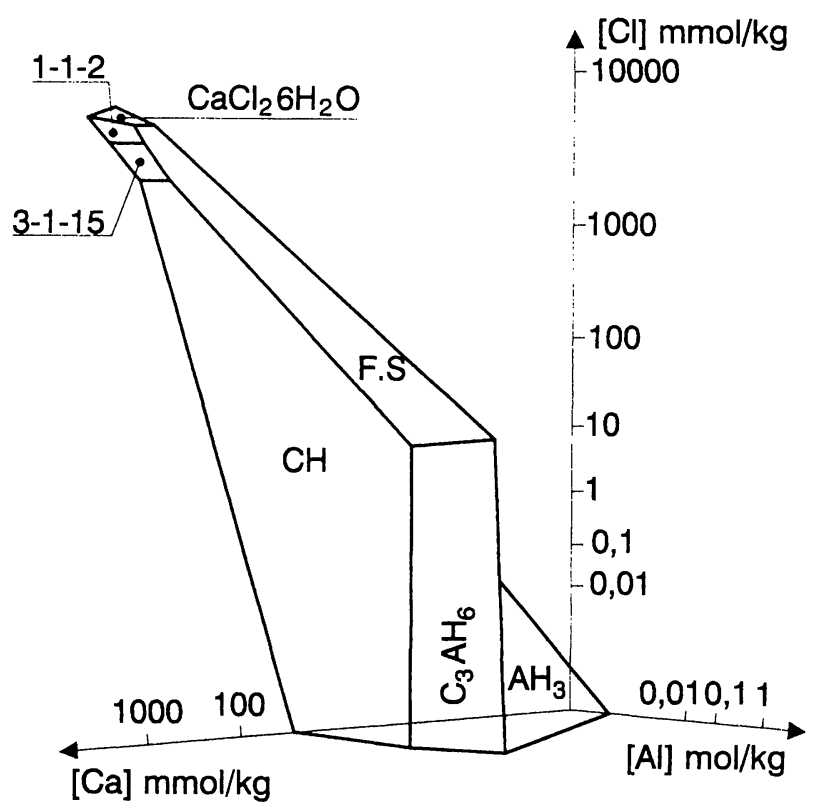

Figura 3.- Representación tridimensional del sistema $\mathrm{CaO}-\mathrm{Al}_{2} \mathrm{O}_{3}-\mathrm{CaCl}_{2}$ $\mathrm{H}_{2} \mathrm{O}$ a $25^{\circ} \mathrm{C}$ (18).Notación: F. S.: sal de Friedel. 3-1-15: $3 \mathrm{CaO} \cdot \mathrm{CaCl}_{2} \cdot 15$ $\mathrm{H}_{2} \mathrm{O} \cdot 1-1-2: \mathrm{CaO} \cdot \mathrm{CaCl}_{2} \cdot 2 \mathrm{H}_{2} \mathrm{O}$. 
que es estable a valores de $\mathrm{pH}$ relativamente bajos y no necesita de $\mathrm{CH}$ (portlandita) para existir. Como vemos en la Figura 3, el monocloroaluminato cálcico tiene una línea de equilibrio con el $3 \mathrm{CaO} \cdot \mathrm{CaCl}_{2} \cdot 15 \mathrm{H}_{2} \mathrm{O}$.

La presencia de álcalis en cl medio, esto es, el sistema $\mathrm{CaO}-\mathrm{Al}_{2} \mathrm{O}_{3}-\mathrm{CaCl}_{2}-\mathrm{Na}_{2} \mathrm{O}-\mathrm{H}_{2} \mathrm{O}$, únicamente varía la localización de los campos de estabilidad del diagrama de fases del sistema $\mathrm{CaO}-\mathrm{Al}_{2} \mathrm{O}_{3}-\mathrm{CaCl}_{2}-\mathrm{H}_{2} \mathrm{O}$, además de añadirse un nuevo campo de estabilidad del $\mathrm{NaCl}$ más o menos perpendicular al eje de cloruros, apareciendo, por tanto, 7 puntos invariantes (Tabla 1 ). A una $\left[\mathrm{Na}^{+}\right]$8,318 $\mathrm{mol} / \mathrm{kg}$ el monocloroaluminato cálcico deja de ser una fase cstable en contacto con una disolución de $\mathrm{NaCl}$. Además, la presencia de álcalis hace que la concentración de cloruros tenga que aumentar para que la sal de Friedel sca una fase cstable. Así, la mínima concentración de cloruros requerida para estabilizar la sal de Friedel pasa de 0,0096 a $0,1049 \mathrm{~mol} / \mathrm{kg}$ (aprox. 10 veces más) y la máxima concentración de cloruros aumenta también de 8,016 a $8,205 \mathrm{~mol} / \mathrm{kg}$ (una variación insignificantc). Además, en este trabajo se hizo, un refinamiento del sistema para bajas y altas concentraciones de cloruro que se explica someramente a continuación:

A bajas concentraciones de cloruro puede haber una sustitución (parcial o total) de $\mathrm{Cl}$ por $\mathrm{OH}^{-}$en el monocloroaluminato cálcico hidratado, para obtener el correspondiente $\mathrm{C}_{4} \mathrm{AH}_{13}$ (o cualquier otro hidrato $\mathrm{C}_{4} \mathrm{AH}_{\mathrm{x}}$ ). Birnin-Yauri y col. (24) demuestran que entre la sal de Friedel y los hidratos AFm existe una familia de disoluciones sólidas, dependiendo de la relación $\mathrm{Cl}^{-} / \mathrm{OH}^{-}$ del medio.
A elevadas concentraciones de cloruros, aparece un nuevo campo de estabilidad (Fig.4) muy pequeño para la cloroetringita, $3 \mathrm{CaO} \cdot \mathrm{Al}_{2} \mathrm{O}_{3} \cdot \mathrm{CaCl}_{2} \cdot 30 \mathrm{H}_{2} \mathrm{O}$. Se sabe que la cloro-etringita es estable a temperaturas inferiores a $0^{\circ} \mathrm{C}$. Por tanto, según estos autores, si la cloro-etringita es estable a $25^{\circ} \mathrm{C}$, su campo de estabilidad únicamente existiría a concentraciones de cloruros muy altas y, por tanto, sólo estaría representando un estado metaestable. Este campo de estabilidad probablemente aumente a medida que la temperatura vaya disminuyendo.

La presencia de este nuevo campo de estabilidad produce la desaparición del punto invariante $\mathrm{AH}_{3}+\mathrm{SF}+$

$\mathrm{CaCl}_{2} \cdot 6 \mathrm{H}_{2} \mathrm{O}$ y la aparición de tres nuevos puntos invariantes:

$\mathrm{CaCl}_{2} \cdot 6 \mathrm{H}_{2} \mathrm{O}+$ cloro-etringita $+\mathrm{SF}$.

$\mathrm{CaCl}_{2} \cdot 6 \mathrm{H}_{2} \mathrm{O}+\mathrm{AH}_{3}+$ cloro-etringita.

$\mathrm{AH}_{3}+\mathrm{SF}+$ cloro-ctringita.

Estos puntos no son estables cuando los cálculos se llevan a cabo en presencia de $0,25 \mathrm{~mol} / \mathrm{kg}$ de $\mathrm{Na}_{2} \mathrm{O}$.

La cloro-ctringita es muy soluble y según dichos autores es casi imposible llegar al equilibrio cuando se redispersa en agua pura ya que toda la cloro-etringita se disuelve. Si la redispersión ocurre en una disolución saturada de $\mathrm{CaCl}_{2}$, la cloro-etringita y la sal de Friedel son fases estables. Sin embargo, a juicio de estos autores, la concentración de aluminio es demasiada baja para ser

TABLA 1

Composiciones de los puntos invariantes de la disolución a $25^{\circ} \mathrm{C}$ para el sistema $\mathrm{CaO}-\mathrm{Al}_{2} \mathrm{O}_{3}-\mathrm{CaCl}_{2}-\mathrm{Na}_{2} \mathrm{O}-\mathrm{H}_{2} \mathrm{O}(18)$.

\begin{tabular}{|c|c|c|c|c|}
\hline Sólidos en equilibrio con la fase acuosa & \begin{tabular}{|l}
$\mathrm{Ca}]$ \\
$\mathrm{mol} / \mathrm{Kg}$
\end{tabular} & $\begin{array}{l}{[\mathrm{Al}]} \\
\mathrm{mol} / \mathrm{Kg}\end{array}$ & $\begin{array}{l}\text { [Na] } \\
\mathrm{mol} / \mathrm{Kg}\end{array}$ & $\begin{array}{l}{[\mathrm{Cl}]} \\
\mathrm{mol} / \mathrm{Kg}\end{array}$ \\
\hline $\mathrm{NaCl}, \mathrm{FS}^{*}, 1-1-2^{* *}, \mathrm{CaCl}_{2} \cdot 6 \mathrm{H}_{2} \mathrm{O}$ & 3.703 & $5.41 \mathrm{E}-11$ & 1.014 & 8.401 \\
\hline $\mathrm{NaCl}, \mathrm{FS}, \mathrm{AH}_{3}, \mathrm{CaCl}_{2} \cdot 6 \mathrm{H}_{2} \mathrm{O}$ & 3.685 & $9.51 \mathrm{E}-8$ & 1.024 & 8.395 \\
\hline $\mathrm{NaCl}, \mathrm{FS}, 1-1-2,3-1-15^{\text {*.ak }}$ & 2.162 & $3.46 \mathrm{E}-11$ & 2.688 & 6.988 \\
\hline $\mathrm{NaCl}, \mathrm{FS}, 3-1-15, \mathrm{CH}$ & $6.32 \mathrm{E}-1$ & $1.80 \mathrm{E}-10$ & 5.081 & 6.333 \\
\hline $\mathrm{NaCl}, \mathrm{FS}, \mathrm{AH}_{3}, \mathrm{C}_{3} \mathrm{AH}_{6}$ & $7.00 \mathrm{E}-7$ & 8.81E-2 & 7.243 & 5.051 \\
\hline $\mathrm{NaCl}, \mathrm{FS}, \mathrm{CH}, \mathrm{C}_{3} \mathrm{AH}_{6}$ & $1.79 \mathrm{E}-5$ & $1.38 \mathrm{E}-3$ & 8.318 & 3.936 \\
\hline $\mathrm{NaCl}, \mathrm{NaOH}, \mathrm{CH}, \mathrm{C}_{3} \mathrm{AH}_{6}$ & $4.08 \mathrm{E}-10$ & $5.12 \mathrm{E}-4$ & 25.11 & $7.87 \mathrm{E}-3$ \\
\hline
\end{tabular}




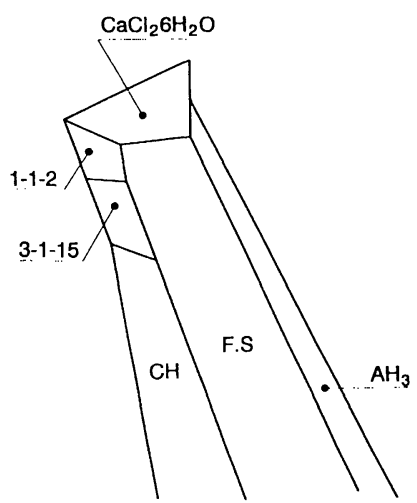

A

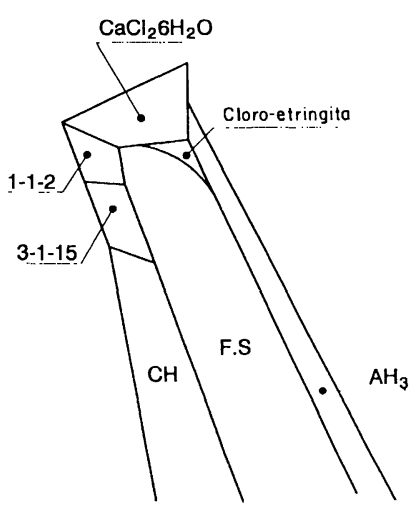

B
Figura 4.- Modificación del sistema CaO- $\mathrm{Al}_{2} \mathrm{O}_{3}-\mathrm{CaCl}_{2}-\mathrm{H}_{2} \mathrm{O}$ a $25^{\circ} \mathrm{C}$, a altas concentraciones de cloruro, debido a la presencia de cloro-etringita (B) y comparado con el descrito anteriormente (A) (18). Notación: F. S.: sal de Friedel; 3-1-15:3 $\mathrm{CaO} \cdot \mathrm{CaCl}_{2} \cdot 15 \mathrm{H}_{2} \mathrm{O} ; 1-1-2: \mathrm{CaO} \cdot \mathrm{CaCl}_{2} \cdot 2 \mathrm{H}_{2} \mathrm{O}$.

analizada con precisión y el dato de solubilidad debe ser utilizado con restricciones a la hora de calcular el producto de solubilidad.

\section{$4.2 \mathrm{El}$ Sistema $\mathrm{CaO}-\mathrm{Al}_{2} \mathrm{O}_{3}-\mathrm{CaSO}_{4}-\mathrm{CaCl} \mathrm{Cl}_{2} \mathrm{O}$. Influencia del $\mathrm{Na}_{2} \mathrm{O}$}

Muchos autores han estudiado como influye la capacidad de fijación de los iones $\mathrm{Cl}^{-}$con los aluminatos de un cemento en presencia de $\mathrm{SO}_{4}{ }^{2-}$ y de iones alcalinos $(2,9$, 17, 25-28). Observaron que a medida que aumenta la cantidad de sulfatos y álcalis en un cemento se produce un efecto inhibidor en la capacidad de fijación de los cloruros. También se ha publicado la existencia de una disolución sólida (26) entre el monocloroaluminato cálcico hidratado y el monosulfoaluminato cálcico hidratado $\left(3 \mathrm{CaO} \cdot \mathrm{Al}_{2} \mathrm{O}_{3} \cdot \mathrm{CaSO}_{4} \cdot 14 \mathrm{H}_{2} \mathrm{O}\right)$, en donde la máxima sustitución es de $\sim 50 \%: 3 \mathrm{CaO} \cdot \mathrm{Al}_{2} \mathrm{O}_{3} \cdot 0,5 \mathrm{CaSO}_{4} \cdot 0,5$ $\mathrm{CaCl}_{2} \cdot \mathrm{nH}_{2} \mathrm{O}$.

Damidot y col. (19) estudiaron el sistema $\mathrm{CaO}-\mathrm{Al}_{2} \mathrm{O}_{3}$ $\mathrm{CaSO}_{4}-\mathrm{CaCl}_{2}-\mathrm{H}_{2} \mathrm{O}$ a $25^{\circ} \mathrm{C}$ y la influencia del $\mathrm{Na}_{2} \mathrm{O}$ en este sistema. Los resultados experimentales de trabajos anteriores $(27,28)$ indicaban que podían darse tres casos, cuando en el medio había sulfatos y cloruros conjuntamente:

- Que la etringita y el monocloroaluminato coexistan juntos.

- Que el monocloroaluminato se transforme en etringita, y

- Que la etringita se convierta en monocloroaluminato.

Los resultados del estudio de Damidot y col. (18) mostraban la presencia de 9 puntos invariantes estables (Tabla 2).

Estos autores, hicieron una representación gcométrica de dicho sistema a temperatura constante, reduciendo las dimensiones de 4 (el agua está en exceso) a 3, fijando la concentración de cloruros. Así, para una $\left[\mathrm{Cl}^{-}\right]$de 0,2

TABLA 2

Composiciones de los puntos invariantes de la disolución a $25^{\circ} \mathrm{C}$ para el sistema CaO- $\mathrm{Al}_{2} \mathrm{O}_{3}-\mathrm{CaCl}_{2}-\mathrm{Na}_{2} \mathrm{O}-\mathrm{H}_{2} \mathrm{O}(19)$.

\begin{tabular}{|l|l|l|l|l|l|}
\hline $\begin{array}{l}\text { Sólidos en equilibrio con la fase } \\
\text { acuosa }\end{array}$ & $\begin{array}{l}{[\mathbf{C a}]} \\
\mathbf{m o l} / \mathbf{K g}\end{array}$ & $\begin{array}{l}{[\mathbf{A l}]} \\
\mathbf{m o l} / \mathbf{K g}\end{array}$ & $\begin{array}{l}{\left[\mathbf{S O}_{4}\right]} \\
\mathbf{m o l} / \mathbf{K g}\end{array}$ & $\begin{array}{l}{[\mathbf{C l}]} \\
\mathbf{m o l} / \mathbf{K g}\end{array}$ & $\mathbf{p H}$ \\
\hline $\mathrm{CH}_{2} \mathrm{C}_{3} \mathrm{AH}_{6}, \mathrm{FS}^{*}$, etringita & $2.59 \mathrm{E}-2$ & $7.18 \mathrm{E}-6$ & $7.29 \mathrm{E}-6$ & $9.58 \mathrm{E}-3$ & 12.50 \\
\hline $\mathrm{AH}_{3}, \mathrm{C}_{3} \mathrm{AH}_{6}, \mathrm{FS}$, etringita & $1.05 \mathrm{E}-2$ & $2.79 \mathrm{E}-4$ & $8.21 \mathrm{E}-6$ & $1.18 \mathrm{E}-2$ & 11.86 \\
\hline $\mathrm{CH}, 3-1-15^{* *}$, yeso, etringita & 1.659 & $2.8 \mathrm{E}-11$ & $4.03 \mathrm{E}-3$ & 3.247 & 11.82 \\
\hline $\mathrm{CH}, 3-1-15, \mathrm{FS}$, etringita & 1.656 & $3.8 \mathrm{E}-10$ & $7.03 \mathrm{E}-4$ & 3.248 & 11.82 \\
\hline $\mathrm{AH}$, yeso, $\mathrm{FS}$, etringita & 2.826 & $4.32 \mathrm{E}-7$ & $1.60 \mathrm{E}-3$ & 5.648 & 9.47 \\
\hline $3-1-15$, yeso, $\mathrm{FS}$, etringita & 2.864 & $5.3 \mathrm{E}-11$ & $1.65 \mathrm{E}-3$ & 5.658 & 11.44 \\
\hline $3-1-15,1-1-{ }^{* * *}$, yeso, FS & 3.250 & $2.7 \mathrm{E}-11$ & $1.25 \mathrm{E}-3$ & 6.426 & 11.34 \\
\hline $\mathrm{AH}_{3}$, yeso, $\mathrm{CaCl}_{2} \cdot 6 \mathrm{H}_{2} \mathrm{O}, \mathrm{FS}$ & 4.001 & $9.56 \mathrm{E}-8$ & $7.04 \mathrm{E}-4$ & 8.001 & 9.07 \\
\hline $1-1-2$, yeso, $\mathrm{CaCl}_{2} \cdot 6 \mathrm{H}_{2} \mathrm{O}, \mathrm{FS}$ & 4.025 & $4.7 \mathrm{E}-11$ & $7.14 \mathrm{E}-4$ & 8.015 & 10.73 \\
\hline
\end{tabular}

" $\mathrm{FS}=$ sal de Friedel o monocloroaluminato cálcico hidratado

$* * * 3-1-15=3 \mathrm{CaO} \cdot \mathrm{CaCl}_{2} \cdot 15 \mathrm{H}_{2} \mathrm{O}$
$\quad 1-1-2=\mathrm{CaO} \cdot \mathrm{CaCl}_{2} \cdot 2 \mathrm{H}_{2} \mathrm{O}$ 
mol/kg (Fig. 5) las fases $\mathrm{AH}_{3}, \mathrm{CH}$, yeso, etringita y monocloroaluminato cálcico hidratado son estables en contacto con la disolución. La etringita y el calcio monocloroaluminato están en equilibrio a lo largo de un rango de composiciones acuosas correspondientes a la línea definida por sus campos de estabilidad.

La etringita es estable en un amplio rango de concentraciones de sulfatos comparado con el monocloroaluminato cálcico hidratado. Sin embargo, a altas concentraciones de cloruros, la estabilidad del monocloroaluminato cálcico hidratado aumenta con respecto a la etringita. Para una $\left[\mathrm{Cl}^{-}\right]=4,5 \mathrm{~mol} / \mathrm{kg}$, la Figura 6 muestra que cl $\mathrm{CH}$ no es estable en contacto con la fase acuosa sino que lo es el $3 \mathrm{CaO} \cdot \mathrm{CaCl}_{2} \cdot 15 \mathrm{H}_{2} \mathrm{O}$.

Comparando ambas figuras, se observa que a medida que aumenta la concentración de cloruro, los campos de estabilidad se van desplazando progresivamente hacia mayores concentraciones de calcio, lo cual es necesario para mantener la neutralidad eléctrica de la disolución, que contiene una elevada cantidad en disolución tanto de $\mathrm{Cl}^{-}$como de $\mathrm{OH}^{-}$. La concentración más alta de cloruros a la cual la etringita es estable es $4,5 \mathrm{~mol} / \mathrm{kg}$.

La conclusión a la que llegaron fue que, efectivamente, el monocloroaluminato cálcico y la etringita pueden coexistir juntas y que dependiendo de la cantidad relativa de sulfato y cloruro presentes en la fase acuosa, la etringita puede transformarse en monocloroaluminato cálcico hidratado o viceversa. La región de cocxistencia de ambos hidratos depende de la concentración en la disolución de cloruro y sulfato.

Se hizo tambićn el estudio de este sistema pero añadiendo sodio en forma de $\mathrm{NaCl}$ y se observó que los constituyentes solubles añadidos al sistema modificaban los equilibrios dominantes pero que a bajas concentraciones no se formaba ningún sólido estable adicional. Cuando la concentración de sodio aumenta, el $\mathrm{NaCl}$ es el primer sólido, con sodio en su composición, que se vuelve estable en el punto invariante: yeso, $\mathrm{NaCl}$, $\mathrm{SF}, \mathrm{CaCl}_{2} \cdot 6 \mathrm{H}_{2} \mathrm{O}, 1-1-2$, agua.

Como consecuencia de esto, cuando la concentración de sodio es inferior a $1 \mathrm{~mol} / \mathrm{kg}$, la formación de fases sólidas en el sistema $\mathrm{CaO}-\mathrm{Al}_{2} \mathrm{O}_{3}-\mathrm{CaSO}_{4}-\mathrm{CaCl}_{2}-\mathrm{Na}_{2} \mathrm{O}-$ $\mathrm{H}_{2} \mathrm{O}$ es comparable con las formadas en el sistema previamente descrito $\mathrm{CaO}-\mathrm{Al}_{2} \mathrm{O}_{3}-\mathrm{CaSO}_{4}-\mathrm{CaCl}_{2}-\mathrm{H}_{2} \mathrm{O}$, esto es, las fases que contienen sodio no son estables. Sin embargo, la adición de sodio modifica la localización de las regiones de las fases. A medida que el sodio aumenta, las disoluciones salinas tienen que estar progresivamente más concentradas en sulfatos y cloruros para estabilizar la etringita y/o la sal de Friedel, respectivamente.

\subsection{Estabilidad frente a la Temperatura}

Se sabe que los cloruros constituyen un peligro para las armaduras del hormigón ya que favorecen la corrosión de las mismas. Quizás, sea menos conocido que algunas sales de cloruro de calcio pueden deteriorar incluso el hormigón (29-31). Esta agresividad se manifiesta por expansiones en el hormigón antes de la aparición de grietas. Particularmente, se observa que, contrariamente a lo que podría ser esperado, la agresividad empicza a ser más severa a bajas temperaturas, $5^{\circ} \mathrm{C}$, mientras que no se observa a temperaturas superiores a $30^{\circ} \mathrm{C}$. Bajo cualquicra de las condiciones citadas anteriormente, se forma el monocloroaluminato cálcico hidratado, pero no se cree que la formación de esta sal o del $\mathrm{Ca}(\mathrm{OH})_{2}$ sean las
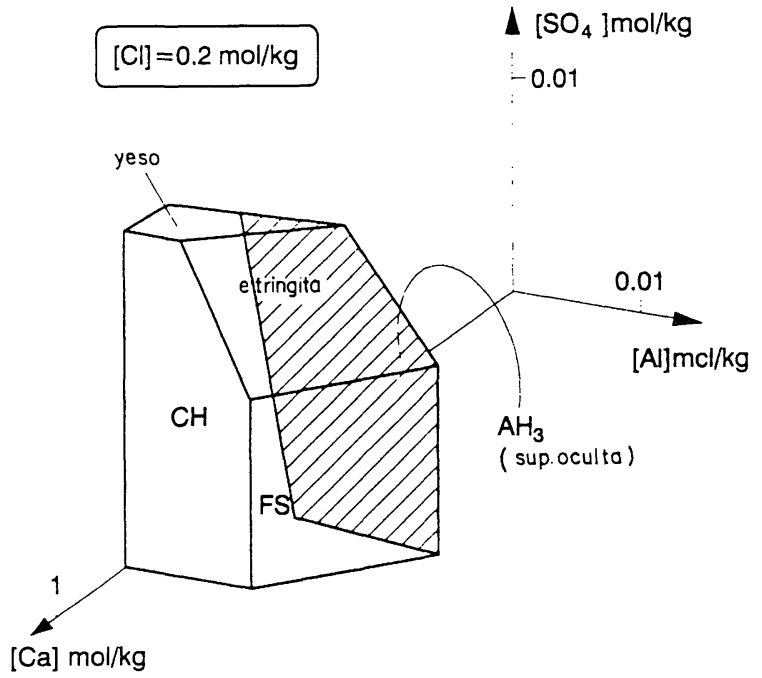

Figura 5.- Representación tridimensional del sistema $\mathrm{CaO}-\mathrm{Al}_{2} \mathrm{O}_{3}-\mathrm{CaCl}_{2}$ $\mathrm{H}_{2} \mathrm{O}$ a $25^{\circ} \mathrm{C}:\left[\mathrm{Cl}^{-}\right]=0,2 \mathrm{~mol} / \mathrm{kg}(19)$. Notación: F. S.: sal de Friedel.

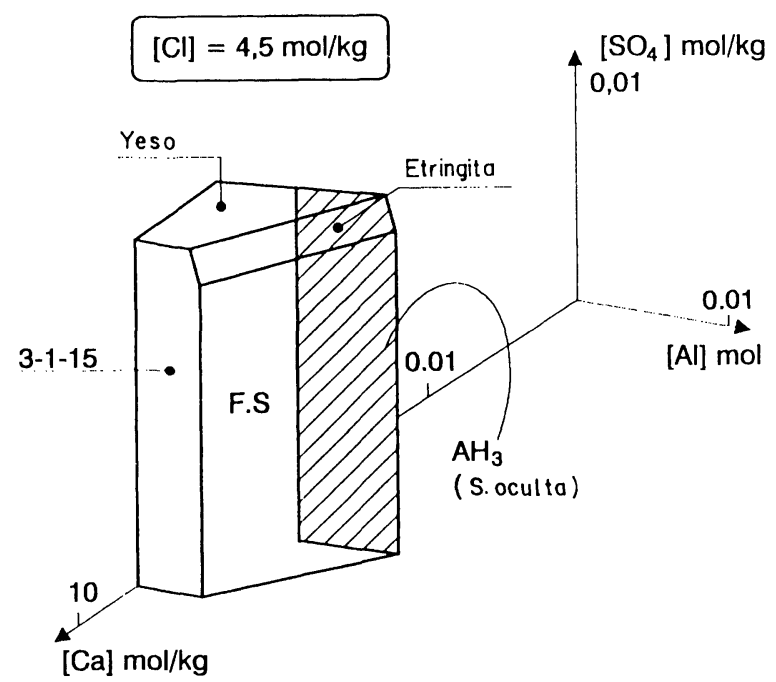

Figura 6.- Representación tridimensional del sistema $\mathrm{CaO})-\mathrm{Al}_{2} \mathrm{O}_{3}-\mathrm{C}^{\mathrm{a}} \mathrm{aCl}_{2}-$ $\mathrm{H}_{2} \mathrm{O}$ a $25^{\circ} \mathrm{C}:\left[\mathrm{Cl}^{-}\right]=4,5 \mathrm{~mol} / \mathrm{kg}$ (19). Notación: F. S.: sal de Friedel; 3-1-15: $3 \mathrm{CaO} \cdot \mathrm{CaCl}_{2} \cdot 15 \mathrm{H}_{2} \mathrm{O}$. 
responsables de la ruptura del cemento Portland, sino que sea debido a la formación de otras sales de cloro como el $3 \mathrm{CaO} \cdot \mathrm{CaCl}_{2} \cdot 15 \mathrm{H}_{2} \mathrm{O}$ o el $\mathrm{CaO} \cdot \mathrm{CaCl}_{2} \cdot 2 \mathrm{H}_{2} \mathrm{O}$.

El monocloroaluminato cálcico hidratado se forma siempre a cualquicr concentración de $\mathrm{CaCl}_{2}$ y en un rango de tempcraturas entre 5 y $40{ }^{\circ} \mathrm{C}$ (29). Sin embargo, a $0{ }^{\circ} \mathrm{C}$ la sal de Friedel con 10 moléculas de agua coexiste con otra sal de 30 moléculas de agua (32 según otros autores), $3 \mathrm{CaO} \cdot \mathrm{Al}_{2} \mathrm{O}_{3} \cdot \mathrm{CaCl}_{2} \mathrm{~S} \cdot 30 \mathrm{H}_{2} \mathrm{O}$, normalmente denominada cloro-ctringita y cuya estabilidad por encima de $0{ }^{\circ} \mathrm{C}$ es incicrta (19), como ya se ha explicado. La cloro-ctringita podría tener a temperaturas inferiores a $0{ }^{\circ} \mathrm{C}$ (además de las dos sales anteriormente mencionadas, $3 \mathrm{CaO} \cdot \mathrm{CaCl}_{2}$. $15 \mathrm{H}_{2} \mathrm{O}$ y CaO$\cdot \mathrm{CaCl}_{2} \cdot 2 \mathrm{H}_{2} \mathrm{O}$ ) propiedades destructivas para el hormigón, como su homóloga sulfatada, la etringita. Más estudios deben realizarse con el objeto de clarificar estos aspectos.

En el lado opuesto, a altas temperaturas se ha de decir que el monocloroaluminato cálcico es estable hasta $85^{\circ} \mathrm{C}$.

\subsection{Estabilidad frente al $\mathrm{pH}$}

Hasta la fecha, no hay evidencias cientificas directas en los artículos publicados que sugieran la ruptura de la sal de Friedel en hormigones contaminados por cloruros durante la vida útil del mismo. La descomposición de la sal de Friedel durante la vida útil de la estructura del hormigón armado, provocaría la liberación de los iones cloruros lo que conllevaría un gran riesgo de corrosión de la armadura. Ello hace necesario que la sal de Friedel tenga que permanecer estable durante la vida útil de una estructura contaminada por cloruros. La sal de Friedel es estable a un $\mathrm{pH}$ relativamente bajo y no necesita de portlandita para existir (ver apartado 4.1). Sin embargo, hay autores que certifican lo contrario y demuestran que la estabilidad de la sal de Friedel presente en hormigones si que parece depender del pH. Así, Suryavanshi y col. (32) hacen un experimento en donde dejan que se forme la sal de Fricdel por ataque a las probetas con $\mathrm{NaCl}$ y posteriormente las someten a un proceso de carbonatación para que disminuya el $\mathrm{pH}$. Observaron, que la sal de Friedel (al igual que la etringita) sólo existía en zonas profundas de la probeta, esto es, donde el frente de carbonatación no había llegado, por lo que, sugirieron que la estabilidad de la sal de Friedel dependía de la alcalinidad que hay entorno al hormigón. Si la alcalinidad disminuye debido a la carbonatación, los cloruros de la sal de Friedel se liberan hacia la disolución del poro. Por tanto, la presencia de un ataque por carbonatación en un hormigón contaminado por cloruros, sufre un alto riesgo de corrosión de las armaduras debido a la descomposición de la sal de Friedel presente. Son necesarias más investigaciones sistemáticas para terminar de esclarecer definitivamente la polémica existente.

\section{IDENTIFICACIÓN DE LA SAL DE FRIEDEL}

La sal de Friedel (monoclínica) presenta su pico más intenso en el difractograma de DRX a un valor $d=7,9 \AA$. Es importante destacar, que tanto la sal de Friedel como su análoga de hierro (III), $3 \mathrm{CaO} \cdot \mathrm{Fe}_{2} \mathrm{O}_{3} \cdot \mathrm{CaCl}_{2} \cdot 10 \mathrm{H}_{2} \mathrm{O}$, aparecen aproximadamente en el mismo espaciado $d$ en el difractograma de DRX, ya que son fases iso-estructurales. Sin embargo, al ser el $\mathrm{C}_{3} \mathrm{~A}$ más reactivo que $\mathrm{el}_{4} \mathrm{AF}$, la sal de Friedel cristaliza preferentemente.

Accidentalmente, el aluminatotetracálcico 13-hidratado, $\mathrm{C}_{4} \mathrm{AH}_{13}$, presenta, también, su pico más intenso $d=7,9 \AA$, lo que hace que la difracción de rayos $\mathrm{X}$ no concluya por si sola la identificación de la sal de Friedel (14).

Por tanto, para confirmar la presencia de la sal de Fricdel se ha de utilizar el Análisis Térmico Diferencial (ATD) que si distingue dicha sal del $\mathrm{C}_{4} \mathrm{AH}_{13}$. La presencia de la sal de Friedel se observa por la aparición de dos picos endotérmicos (Fig. 7) cuyo valor varía según los autores. El primer pico aparece centrado a $\sim 133^{\circ} \mathrm{C}$ (14) ó $\sim 120^{\circ} \mathrm{C}(24)$ y se debe a la climinación de agua entre capas, obteniéndose un producto poco cristalino que se atribuye al $3 \mathrm{Ca}(\mathrm{OH})_{2} 2 \mathrm{Al}(\mathrm{OH})_{3} \mathrm{CaCl}_{2}$. Esta fase persiste hasta $-320^{\circ} \mathrm{C}(14)$ ó $\sim 290{ }^{\circ} \mathrm{C}$ (24) ó $350{ }^{\circ} \mathrm{C}$ (33) donde aparece un segundo pico endotérmico debido a la pérdida del resto del agua, dando un producto totalmente amorfo. Este producto recristaliza exotermicamente a $\sim 670{ }^{\circ} \mathrm{C}$, dando principalmente cloroaluminato cálcico, $11 \mathrm{CaO}$. $7 \mathrm{Al}_{2} \mathrm{O}_{3} \cdot \mathrm{CaCl}_{2}$.

El espectro de infrarrojo por transformada de Fourier, FTIR, es también una buena técnica para identificar la sal

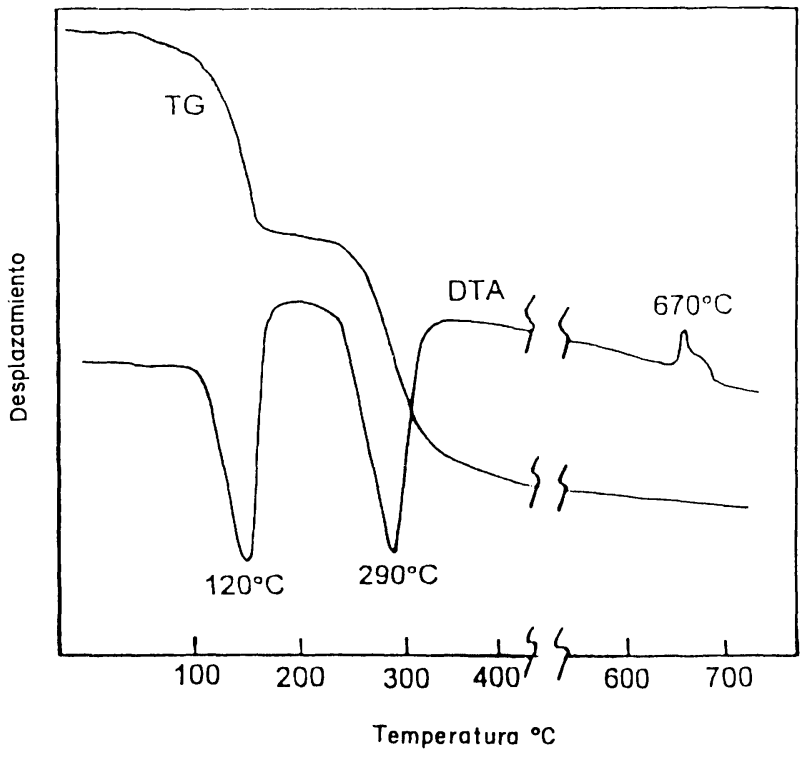

Figura 7.- ATI/TG combinado de la sal de Friedel pura (24). 
de Friedel (Fig.8). Las bandas más características son: la banda a $786 \mathrm{~cm}^{-1}$ debida a la vibración de deformación $\mathrm{Al}$ $\mathrm{O}-\mathrm{H}$. Las bandas en 1.623 y $3.495 \mathrm{~cm}^{-1}$ se asignan a las vibraciones típicas del agua y las bandas que aparecen entre 400 y $600 \mathrm{~cm}^{-1}$ son debidas a las tensiones Al-O (24, $34,35)$. Los cloruros no absorben en el rango de 400$4000 \mathrm{~cm}^{-1}$ ya que no están directamente enlazados al aluminio, por lo que la estructura se puede simplificar como $2 \times\left(\mathrm{Ca}_{2}\left[\mathrm{Al}(\mathrm{OH})_{6}\right] \mathrm{Cl} \cdot 2 \mathrm{H}_{2} \mathrm{O}\right)$.

El espectro de Resonancia Magnética Nuclear de ${ }^{27} \mathrm{Al}$ de la sal de Friedel muestra un único pico a un desplazamiento químico aproximado de $9 \mathrm{ppm}$, característico del aluminio en coordinación octaédrica.

\section{AGRADECIMIENTOS}

El primer autor aprovecha esta ocasión para agradecerle a la Consejería de Educación y Cultura de la Comunidad
Autónoma de Madrid, la beca para la Formación de Personal Investigador que le fucra concedida.

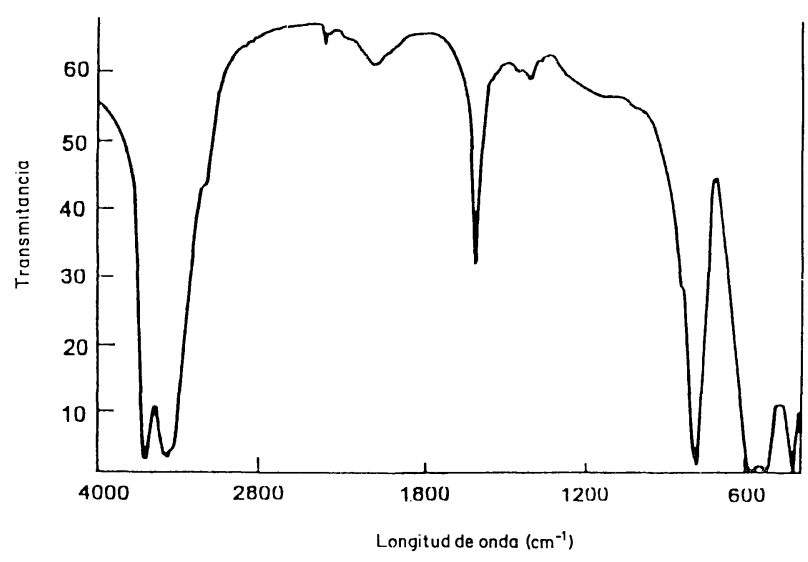

Figura 8.- Espectro de infrarrojo por transformada de Fourier, FTIR, de la sal de Friedel pura (24).

\section{BIBLIOGRAFÍA}

(1) P.M. Friedel: Sur un chloro-aluminate de calcium hydraté se maclant par compression. Bull. Soc. Franc. Minéral, 19(1897), pp. $122-36$. (2) M.H. Roberts: Effect of calcium chloride on the durability of pre-tensioned wire in prestressed concrete. Magazine of Concrete Research, 14(1962),pp. 143-154.

(3) A. Kumar, S.Komarneni y D.M. Roy: Diffusion of $\mathrm{Cs}^{+}$and $\mathrm{Cl}^{-}$through sealing materials. Cem. Concr. Res. 17 (1987), pp. 153-160.

(4) J. Tritthart: The influence of the hydroxide concentration in the pore solution of hardened cement paste on chloride binding. Cem. Concr. Res. 19(1989), pp. 683-691.

(5) H.F.W.Taylor: Cement Chemistry, p. 173. Academic Press, London (1990).

(6) Rasheeduzzafar, S.S. Al-Saadoun, A.S. Al-Gahtani y F.H. Dakhil: Effect of tricalcium aluminate content of cement on corrosion of reinforcing steel in concrete. Cem. Concr. Res. 20 (1990), pp. 723-738.

(7) C. Arya, N.R. Buenfield y J.B. Newman: Factors influencing chloride-binding in concrete. Cem. Concr. Res. 20 (1990), pp. $291-300$.

(8) Rasheeduzzafar, S. Ehtesham Hussain y S. S. Al-Saadoun: Effect of cement composition on chloride binding and corrosion of reinforcing steel in concrete. Cem. Concr. Res. 21 (1991), pp. 777-794.

(9) R. Duval: La Durabilité des Bétons. Presses Ponts et chaussées, Paris, p.196 (1992).

(10) A.Y. Talib, Rasheeduzzafary A.S. Al-Gahtani: Chloride binding and corrosion in silica fume concrete. Concrete 2000 Economic and durable construction through excellence. Proceedings of the International Conference (Scotland, UK) (1993), pp. 1453-1466.

(11) P.J.C. Bloem and B.J.G. Sciarone: Effect of fly ash in concrete on the migration of chlorides. Fifth CANMET/ACI International Conference on Fly Ash, Silica Fume, Slag and Natural Pozzolans in Concrete. Supplementary papers. (1995), Milwaukee, USA, pp. 305-327.

(12) C. Arya y Y. Xu: Effect of cement type on chloride binding and corrosion of steel in concrete. Cem. Concr. Res. 25 (1995), pp. 893902.

(13) A.K. Suryavanshi, J.D. Scantlebury y S.B. Lyon: Corrosion of reinforcement steel embedded in high water-cement ratio concrete contaminated with chloride. Cem. Concr. Comp., 20 (1998), pp. 263-381.

(14) A.K. Suryavanshi, J.D. Scantlebury y S.B. Lyon: The binding of chloride ions by sulphate resistant Portland cement. Cem. Concr. Res. 25(1995),pp. 581-592.

(15) R.K. Dhir, M.A.K. El-Mohry T.D. Dyer: Chloride binding in GGBS concrete. Cem. Concr. Res. 26, (1996), pp. 1767-1773.

(16) R. Mejia Rentería: Contribución al estudio analítico y fisicoquímico del sistema: Cementos Portland, puzolanas y escoria siderúrgica, cloruros y agua. Tesis Doctoral. Universidad Complutense de Madrid. Ftad. Ciencias Químicas (1997).

(17) S. Ehtesham Hussain, Rasheeduzzafar y A.S. Al-Gahtani: Influence of sulfates on chloride binding in cements. Cem. Concr. Res., 24 (1994),pp. 8-24.

(18) D. Damidot, U.A. Birnin-Yauri y F.P. Glasser: Thermodynamic investigation of the $\mathrm{CaO}-\mathrm{Al}_{2} \mathrm{O}_{3}-\mathrm{CaCl}_{2}-\mathrm{H}_{2} \mathrm{O}$ system at $251 \mathrm{C}$ and the influence of $\mathrm{Na}_{2} \mathrm{O}$. Il Cemento. 4(1994), pp. 243-254.

(19) D. Damidot y F.P. Glasser: Thermodynamic investigation of the $\mathrm{CaO}-\mathrm{Al}_{2} \mathrm{O}_{3}-\mathrm{CaSO}_{4}-\mathrm{CaCl}_{2}-\mathrm{H}_{2} \mathrm{O}$ system at $251 \mathrm{C}$ and the influence of $\mathrm{Na}_{2} \mathrm{O}$. Proceedings of the $10^{\text {th }}$ International Congress on the Chemistry of Cement (Gothenburg, Sweden). 4 (1997), 4 iv066.

(20) A.K. Suryavanshi, J.D. Scantlebury y S.B. Lyon: Mechanism of Friedel's salt formation in cements rich in tri-calcium aluminate. Cem. Concr. Res. 26(1996), pp. 717-727.

(21) G. Renaudin, F. Kubel, J.-P. Rivera y M. Francois: Structural phase transition and high temperature phase structure of Friedel's salt, $3 \mathrm{CaOAl}_{2} \mathrm{O}_{3} \mathrm{CaCl}_{2} \mathrm{H}_{2} \mathrm{O}$. Cem. Concr. Res. 29 (1999), pp. 1937-1942. 
(22) M. Ben-Yair: The effect of chlorides on concrete in hot and arid regions. Cem. Concr. Res. 4 (1974), pp. 405-416.

(23) I.G. Richardson y G.W. Groves: The incorporation of minor and trace elements into calcium silicate hydrate (C-S-H) gel in hardened cement pastes. Cem. Concr. Res. 23 (1993), pp. 131-138.

(24) U.A. Birnin-Yauriy F.P. Glasser: Friedel's salt, $\mathrm{Ca}_{2} \mathrm{Al}(\mathrm{OH})_{6}(\mathrm{Cl}, \mathrm{OH}) 2 \mathrm{H}_{2} \mathrm{O}$ : its solutions and their role in chloride binding. Cem. Concr. Res. 28(1998),pp. 1713-1723.

(25) W.R. Holden, N.R. Short y C.L. Page: Corrosion of Reinforcement in Concrete Construction. pp. 143-150. Ellis Horwood Publishers, London(1983).

(26) Y. Tady y B. Fritz: An ideal solid solution model for calculating solubility of clay minerals. Clay Minerals, 16(1981),pp. 361-373.

(27) C. Ftikos, V. Kasselouris, S. Tsimas y G. Parissakis: $\Lambda$ study on the action of sea water on hydrated cement pastes. $7^{\text {th }}$ International Congress on the Chemistry of Cement. Paris. 4 (1980), pp.357-362.

(28) F. Zhang, Z. Zhou y Z. Lou: Solubility product and stability of ettringite. $7^{\text {th }}$ International Congress on the Chemistry ofCement. Paris. 2(1980),pp.88-93.

(29) S. Chatterji: Mechanism of the $\mathrm{CaCl}_{2}$ attack on Portland cement concrete. Cem. and Concr. Res. 8 (1978), pp. 461-468.

(30) S. Monosi, I Alverà y M. Collepardi: Chemical attack of calcium chloride on the Portland cement paste. Il Cemento. 2 (1989), pp. 97104.

(31) S. Manosi y M. Collepardi: Research on $3 \mathrm{CaOCaCl}_{2} 15 \mathrm{H}_{2} \mathrm{O}$ identified in concretes damaged by $\mathrm{CaCl}_{2}$ attack. Il Cemento. 1 (1990), pp. 3-8.

(32) A.K. Suryavanshiy R. Narayan Swamy: Stability of Friedel's salt in carbonated concrete structural elements. Cem. Concr. Res. 26(1996), pp. 729-741.

(33) C.L. Page, N.R. Short y El Tarras: Diffusion of chloride ions in hardened cement pastes. Cem. Concr. Res. 11 (1981), pp. $395-406$.

(34) J. Bensted: Chloroaluminates and the role of calcium chloride in accelerated hardening of Portland cement. World Cement Technology. 8(1977), pp. 171-175.

(35) P. Tarte: Infra-red spectra of inorganic aluminates and characteristic vibrational frequencies of $\mathrm{AlO}_{4}$ tetrahedra and $\Lambda 1 \mathrm{O}_{6}$ octahedra. Spectrochimica Acta. 23A (1967), pp. 2127-2143. 